\title{
Gibt es (noch) eine deutschsprachige Politische Geographie?
}

\author{
B. Korf \\ Geographisches Institut, Universität Zürich, Zürich, Switzerland \\ Correspondence to: B. Korf (benedikt.korf@geo.uzh.ch)
}

Reuber, P.: Politische Geographie, UTB Schöningh, Stuttgart, 266 pp., ISBN-13: 978-3825284862, €29.99, 2012.

Als ich vor einigen Jahren nach einem deutschsprachigen Lehrbuch für meine neue Einführungsveranstaltung zur Politischen Geographie suchte, fand ich nur das klassische Lehrbuch von Klaus-Achim Boesler aus dem Jahr 1983! Dabei hatten die Politische Geographie und die (kritische) Geopolitik im deutschsprachigen Raum seit der Jahrtausendwende eine durchaus bemerkenswerte Renaissance durchlebt und auch einige fundamentale Paradigmenwechsel in Folge des cultural turn vollzogen. Die Konsultation angloamerikanischer Lehrbücher konnte diese Lücke nur unzureichend ausfüllen, weil dort viele empirische Beispiele und die Rezeption der Forschung einseitig auf die Bedürfnisse, Kenntnisse und Weltbilder von britischen oder amerikanischen Studierenden ausgerichtet sind.

Umso mehr sollten wir dem Autor dieses neuen Lehrbuches danken, dass nun wieder ein zeitgemässes Lehrbuch zu diesem Teilgebiet der Humangeographie vorliegt, das einen hervorragenden Einblick in den Stand der Forschung im Fach Politische Geographie bietet. Paul Reuber legt einen umfassenden Überblick über die unterschiedlichen Ansätze der deutschsprachigen Politischen Geographie vor und verortet diese deutschsprachigen Beiträge im Resonanzraum der ,internationalen“ - anglo-amerikanischen - Geographie.

Dabei ist es Reuber besonders hoch anzurechnen, dass er sich für einen Ansatz entschieden hat, der dem Facettenreichtum und der Vielfalt der Politischen Geographie Raum gibt. Auch wenn die spezifischen Vorlieben und die theoretische Verortung des Autors aus seinen Schriften bekannt ist, schreibt Reuber gerade nicht eine teleologische Fachgeschichte, die in einem überlegenen Endpunkt eines dominierenden Paradigmas (meist demjenigen des Autors selbst) endet, wie man dies oft in anderen Lehrbüchern lesen kann. Vielmehr zeigt Reuber auf, welche Dinge, Phänome- ne, Strukturen oder Prozesse der jeweilige Ansatz sichtbar macht - und welche durch diesen Ansatz eher verdeckt werden. Auch veranschaulicht Reuber die einzelnen Ansätze mit empirischen Beispielen, in denen die Herangehensweise und die grundlegenden Erkenntnisse und Aussagen deutlich werden, und formuliert eine Liste von Forschungsfeldern, in denen derzeit die Forschungsfronten lokalisiert werden können. Manche mögen diesen „Eklektizismus“ eher kritisch sehen, ich finde ihn für ein Lehrbuch durchaus hilfreich und überzeugend.

Ich möchte im Folgenden weniger auf einzelne Kapitel des Buches eingehen. Stattdessen möchte ich Reuber's Darstellung des Faches und seiner verschiedenen Denkschulen zum Anlass nehmen, einige Gedanken über den ( $\mathrm{Zu}$-) Stand der Politischen Geographie im deutschsprachigen Raum zu formulieren. Dabei werde ich mich von der Frage leiten lassen, ob es angesichts der zunehmenden Internationalisierung der akademischen Welt überhaupt noch eine deutschsprachige Politische Geographie gibt - oder geben sollte.

Beim Lesen des Kapitels zu Radical Geography wird diese Frage besonders augenfällig - an der Bezeichnung des Forschungsansatzes, der ja nicht „Radikale Geographie“ genannt, sondern mit explizitem Bezug zur angloamerikanischen Debatte als "radical geography" betitelt wird. Deutschsprachige radical geography - ist das nicht ein Widerspruch? Reuber löst diesen Widerspruch auf, indem er diejenigen Schriften, die von deutschsprachigen Geographinnen an deutschen (oder Schweizer oder österreichischen) Hochschulen - vornehmlich auf deutsch - publiziert werden, in diesem Kapitel abhandelt, gleichzeitig aber auch den internationalen Debattenkontext rezipiert. Diese radical geography ist inspiriert durch Autoren wie David Harvey, Neil Smith, Neil Brenner; Richard Peet und deren (post-) marxistische Schriften - eine Denkschule, die sich im anglo-amerikanischen Raum explizit gegenüber einer poststrukturalistischen „kritischen“ Geographie abgrenzt. 
Problematisch finde ich an dieser deutschsprachigen radical geography eine recht einseitige Orientierung an angloamerikanischen „Helden“ was wiederum die Frage aufwirft, warum kontinentaleuropäische und insbesondere deutschsprachige (post-) marxistische Denkschulen so wenig zur Kenntnis genommen und rezipiert werden - gerne auch kritisch: z.B. die neueren Schriften aus der Frankfurter Schule, Axel Honneth's Theorie der Anerkennung oder Rainer Forst's Kritik der Rechtfertigungsverhältnisse.

Dieses Problem bleibt nicht auf die radical geography begrenzt - dort wird es durch die (Selbst-) Bezeichnung nur besonders evident. Auch in der poststrukturalistischen Politischen Geographie oder in den Schriften zur kritischen Geopolitik zeigt sich ein Trend zur primären Rezeption angloamerikanischer Theoriedebatten, die dann als ,international“ den anscheinend ,verstaubten nationalen“ Forschungsfeldern als eine Art Modernisierungsmedizin verabreicht werden. Ich möchte nicht bestreiten, dass dies einmal notwendig erschien angesichts des eher marginalen und theoriefernen Zustands der deutschsprachigen Politischen Geographie vor dem cultural turn. Aber manchmal würde ich mir eine kritische Reflektion über die anglo-amerikanische Rezeption kontinentaleuropäischer Theoretiker und Philosophinnen wünschen. Die umstrittene Rezeption von Henry Lefebvre durch amerikanische Geographen wie Ed Soja wird zum Beispiel dann problematisch, wenn Lefebvre überwiegend durch Soja et al.'s Lesart im deutschsprachigen Raum Eingang in die Theoriediskussion findet. Auch andere französische poststrukturalistische Theoretiker scheinen erst dann im deutschsprachigen Raum zur Kenntnis genommen zu werden, wenn sie in der anglo-amerikanischen Diskussion den Durchbruch geschafft haben.

In der Diskussion über „,das Politische“ und seine Abgrenzung zu Politik werden vornehmlich französisch-sprachige Autoren, wie Jacques Rancière, rezipiert. Auch hier tut sich eine überraschende Leerstelle auf: Carl Schmitt und seine Definition des Politischen als Freund-Feind-Unterscheidung ist in der deutschsprachigen Politischen Geographie irgendwie übersehen worden. Erst als die Schmitt-Rezeption in der anglo-amerikanischen Geographie forciert wurde, kam es in der deutschsprachigen Geographie zu einer zögerlichen Beschäftigung mit seinen Arbeiten. Sicher, eine Rezeption ist aufgrund der Schmitt'schen Apologetik des nationalsozialistischen Reiches nicht unproblematisch, was aber einer - kritischen - Auseinandersetzung mit seinem Werk nicht grundsätzlich im Wege stehen sollte, gerade auch, weil der Begriff des Raumes so zentral in Schmitt's Denken ist.

Vielmehr noch: mir scheint die neue Mode der SchmittRezeption im anglo-amerikanischen Kontext - innerhalb wie ausserhalb der Geographie - teilweise etwas kontextentbunden, man könnte auch sagen, in der Tendenz zu wenig kritisch, zu affirmativ, zu wenig den historischen Entstehungskontext der Schmitt'schen Schriften reflektierend. Genau hier könnte die Aufgabe der deutschsprachigen Politischen Geographie liegen, eine kritische Rezeption Schmitt'scher
Schriften einzufordern und seine Raumtheorie auf ihre heutige Alltagstauglichkeit zu testen statt einige süffisante Aussagen und prägnante Sätze (à la ,Souverän ist, wer über den Ausnahmezustand entscheidet"), von denen Schmitt recht viele in seinem Werk verteilt hat, assoziativ $\mathrm{zu}$ verwerten, wie dies oft in der anglo-amerikanischen Diskussion geschieht.

Auf der anderen Seite gibt es in der deutschsprachigen Tradition einige Denkschulen, die sich weitgehend abgekoppelt von anglo-amerikanischen Denkschulen entwickelt haben. Die Systemtheorie Luhmann'scher Prägung ist sicher der eindeutigste Fall (sie wird im Lehrbuch nicht weiter vertieft). Dies bedeutet nicht notwendigerweise, dass diese deutschsprachigen Traditionen nicht auch in Auseinandersetzung mit Theoretikerinnen aus nicht-deutschsprachigen Traditionen entwickelt wurden (Luhmann grenzte sich zum Beispiel vom amerikanischen Soziologen und Systemtheoretiker Talcott Parsons ab); der springende Punkt ist, dass diese Theoriedebatten innerhalb der deutschsprachigen Geographie ohne grundlegende Auseinandersetzung oder Rezeption der anglo-amerikanischen Theoriedebatte in der Humangeographie entwickelt wurden und als Folge an die sogenannte ,internationale“ Diskussion wenig anschlussfähig sind was nicht gegen diese Theorien spricht. Es wäre sicher interessant, Luhmann'sches Gedankengut einmal in Konversation mit der anglo-amerikanischen Faszination für Latour zu bringen (vgl. Lippuner, 2009), dann aber eben im Konversationsraum ,internationaler“ Geographie. Das könnte aus meiner Sicht gerade zu einer kosmopolitischen Öffnung angloamerikanischer Selbstgespräche beitragen.

Es gibt auch Situationen, in denen theoretische Impulse des cultural turn aus der anglo-amerikanischen Geographie in der deutschsprachigen Politischen Geographie aufgegriffen und innovativ weiterentwickelt oder systematisiert wurden. So scheint mir die Entwicklung lexikometrischer Verfahren der Diskursanalyse eine Spezialität deutscher Gründlichkeit zu sein, die die „Subjektlosigkeit“ des Foucault'schen Diskursbegriffs methodologisch abzubilden versucht (Dzudzek et al., 2010). Mathew Hannah, einer der wenigen anglo-amerikanischen Autoren, die sehr gut mit der deutschsprachigen Geographie vertraut sind, lobt deshalb auch die ,,vorsichtigere und differenziertere Rezeption des Poststrukturalismus“ im deutschsprachigen Raum (Hannah, 2011:55). Dies wirft die Frage auf: Wie könnte man solche Neuerungen, die am Rande oder ausserhalb des angloamerikanischen Mainstream entstanden sind, stärker in eine internationale Debatte zur humangeographischen Rezeption von Diskurstheorien einbringen?

Vielleicht ist es ein bisschen das Problem von Hase und Igel: Wenn mit zeitlicher Verzögerung im deutschsprachigen Raum anglo-amerikanische Theoriedebatten aufgegriffen werden (böse Zungen sprechen von eimem 10 years $t$ me lag, der sich aber, so scheint mir, in letzter Zeit deutlich verkürzt hat), so haben diese in der schnelllebigen Welt anglo-amerikanischer Theoriemoden meist ihre 
innovative Phase bereits hinter sich; die Theoriekaravane ist schon weitergezogen. Dies zeigt sich auch im vorliegenden Lehrbuch, in dem Diskurstheorie und Gouvernementalitätsansätze als neueste Theorieschulen einer poststrukturalistischen deutschsprachigen Politischen Geographie dargestellt werden - und richtig so, denn dies spiegelt den derzeitigen Stand dort wider. In der anglo-amerikanischen Geographie hat sich die Theoriedebatte schon umorientiert und am sogenannten materialist turn festgebissen - mit Debatten zu einer Theorie des Affektes (also gerade der prä-kognitiven und prä-diskursiven Sphären), non-representational theory und zu Latour's Akteursnetzwerktheorie, oder jüngst auch: assemblage theory mit Deleuze, die alle in Spannung zu Foucault's Vermächtnis stehen. Erste Ansätze einer Rezeption dieser Theorien sind mittlerweile auch im deutschsprachigen Kontext zu erkennen, doch eher als einfache Übertragung denn in kritischer Annäherung. Und gleichzeitig wäre zu fragen: sind die Potentiale und Probleme der Diskurstheorie bereits ausreichend ausgelotet, dass man sie schon hinter sich lassen und auf die nächste Theoriemode aufspringen kann?

Ich habe diese Fragen aufgeworfen, um eine viel grundlegendere zu stellen: Wie könnte eine kosmopolitische Politische Geographie aussehen und welche Rolle könnte dabei der deutschsprachigen Tradition zukommen? Zugegebenermassen gibt es dafür vorerst keine einfache Antwort. Ich denke aber, dass ein Weg über eine bewusstere Pflege bestimmter deutschsprachiger „Besonderheiten“ in der Politischen Theorie und Philosophie (zum Beispiel die neuere Generation der Frankfurter Schule, aber auch: Carl Schmitt's Erbe) und Gesellschaftstheorie (zum Beispiel Niklas Luhmann) führen könnte - aber nur, wenn dieses Erbe nicht allein im deutschsprachigen Kontext bearbeitet, sondern selbstbewusst in einem internationalen, englisch-sprachigen Debattenraum eingebracht wird.
Literatur

Boesler, K.-A.: Politische Geographie, Teubner, Stuttgart, 1983.

Dzudzek, I., Glasze, G., Mattissek, A., und Schirmel, H.: Verfahren der lexikometrischen Analyse von Textkorpora, in: Handbuch Diskurs und Raum, Herausgeber: Glasze, G. und Mattissek, A., Transcript, Bielefeld, 233-260, 2010.

Hannah, M.: Zum anti-deterministischen Geist in der Geographie, Geogr. Helv., 66, 54-59, 2011.

Lippuner, R.: Hybridität und Differenz. Zur (Neu-) Thematisierung der materiellen Welt in der Humangeographie, Berichte zur deutschen Landeskunde, 83, 143-161, 2009. 